\title{
Pulmonary Rehabilitation Improves Subjective Sleep Quality in COPD
}

\author{
Chou-Chin Lan MD PhD, Hui-Chuan Huang MSc, Mei-Chen Yang MD, \\ Chih-Hsin Lee MD PhD, Chun-Yao Huang MD, and Yao-Kuang Wu MD
}

\begin{abstract}
BACKGROUND: Poor sleep quality is often reported among patients with COPD. Pulmonary rehabilitation (PR) is beneficial in improving exercise capacity and health-related quality of life (HRQOL). However, its benefit in terms of sleep quality in patients with COPD remains unclear. This study aimed to investigate the effects of PR on sleep quality of patients with COPD. METHODS: Thirty-four subjects with COPD were studied. All subjects participated in a 12-week (2 sessions/ week) hospital-based out-patient PR study. Baseline and post-PR status were evaluated by spirometry, a sleep questionnaire (Pittsburgh Sleep Quality Index [PSQI]), a disease-specific questionnaire of HRQOL (St George Respiratory Questionnaire [SGRQ]), cardiopulmonary exercise testing, respiratory muscle strength, and the Borg dyspnea scale. RESULTS: Mean FEV $_{1} / \mathrm{FVC}$ in the subjects was $0.49 \pm 0.13$, and the mean $\mathrm{FEV}_{1}$ was $1.06 \pm 0.49 \mathrm{~L} / \mathrm{min}(49.7 \pm 18.0 \%$ of predicted $)$. After PR, the PSQI score decreased from $9.41 \pm 4.33$ to $7.82 \pm 3.90(P<.001)$. The number of subjects with a PSQI score $>5$ also decreased $(85.3-64.7 \%, P=.006)$. There were significant improvements in HRQOL (SGRQ, $P=.003$ ), exercise capacity (peak oxygen uptake, $P<.001$; and work rate, $P<.001)$, dyspnea score $(P<.001)$, and respiratory muscle strength (inspiratory muscle strength, $P=.005$; and expiratory muscle strength, $P=.004)$ after $P R$. There were no significant changes in pulmonary function test results $\left(\mathrm{FEV}_{1}, P=.77 ; \mathrm{FVC}, P=.90 ; \mathrm{FEV}_{1} / \mathrm{FVC}, P=.90\right)$. CONCLUSIONS: PR results in significant improvement in sleep quality, along with concurrent improvements in HRQOL and exercise capacity. PR is an effective nonpharmacologic treatment to improve sleep quality in patients with COPD and should be part of their clinical management. Key words: COPD; health-related quality of life; pulmonary rehabilitation; sleep quality. [Respir Care 2014;59(10):1569-1576. (C) 2014 Daedalus Enterprises]
\end{abstract}

\section{Introduction}

Poor sleep quality is often reported among patients with COPD. ${ }^{1}$ They frequently complain of difficulty initiating and maintaining sleep and an increased number of arousals during sleep. ${ }^{2}$ A previous polysomnographic study demonstrated low total sleep time, sleep fragmentation, and reduced amounts of deep sleep in patients with COPD. ${ }^{1} \mathrm{In}$

\footnotetext{
The authors are affiliated with the Division of Pulmonary Medicine, Taipei Tzu Chi Hospital, Buddhist Tzu Chi Medical Foundation, New Taipei City, Taiwan and the School of Medicine, Tzu Chi University, Hualien, Taiwan, with the exception of Miss H-C Huang, who is affiliated with the Department of Nursing, Cardinal Tien College of Healthcare and Management, New Taipei City, Taiwan.
}

The authors have disclosed no conflicts of interest. previous studies, $61-75 \%$ of patients with COPD had poor sleep quality. ${ }^{2-4}$ Another study suggested that poor sleep quality happens in these patients regardless of Global Initiative for Chronic Obstructive Lung Disease (GOLD) classification of COPD. ${ }^{4}$ Even those with mild-to-moderate COPD have poor sleep quality. ${ }^{5}$ However, poor sleep quality is frequently ignored by physicians in the management of COPD. Despite the apparent high prevalence of night-

\footnotetext{
Correspondence: Dr Yao-Kuang Wu, Division of Pulmonary Medicine, Taipei Tzu Chi Hospital, Buddhist Tzu Chi Medical Foundation, No. 289, Jianguo Road, New Taipei City 23142, Taiwan. E-mail: drbfci@ yahoo.com.tw.
}

DOI: $10.4187 /$ respcare. 02912 
time symptoms and disturbed sleep, there are only a few interventional studies that have directly targeted them.

Sleep is an important aspect of maintaining the body's circadian rhythm. Inadequate sleep has impact on longterm health consequences, and sleep disturbance is associated with cardiovascular risk factor abnormalities, type 2 diabetes mellitus, fatigue, lethargy, depression, impaired cognition, and overall poor quality of life..$^{2,6-8}$ Because poor sleep quality contributes to the increased comorbidities and impact on general health, there should be increased efforts to treat poor sleep quality in COPD. Hypnotics should be avoided because of a potential deleterious effects on respiration during sleep. ${ }^{9}$ Instead, the management of sleep problems in COPD should first focus on optimizing the patient's overall respiratory condition. Previous studies showed equivocal benefits of theophylline, inhaled bronchodilators, and supplemental oxygen on sleep quality. ${ }^{10-15}$ Management should therefore not just concentrate on drug control of airway obstruction.

Subjective sleep quality can be assessed via questionnaire, clinical interviews, and sleep diaries, ${ }^{16}$ whereas objective sleep quality can be obtained by polysomnography and actigraphy. ${ }^{16}$ Although overnight in-laboratory polysomnography is the accepted standard for diagnosing sleep disorders, it is expensive and burdensome for patients. Furthermore, in-laboratory polysomnography may not reflect usual sleep conditions. Sleep is largely subjective in nature. Subjective sleep quality as assessed by a questionnaire provides useful insights into the patient's perception of the nocturnal burden. In a previous study, symptoms of depression were strongly associated with subjective sleep quality and only moderately associated with objective sleep quality. ${ }^{17}$ In the current study, the Pittsburgh Sleep Quality Index (PSQI) was used to evaluate subjective sleep quality in patients with COPD.

Pulmonary rehabilitation (PR) is often recommended as an integral part of management, ${ }^{18,19}$ and many studies confirm that with PR there is improvement in exercise capacity and health-related quality of life (HRQOL) in patients with COPD. ${ }^{20-22}$ Despite numerous studies confirming that PR improves HRQOL and exercise capacity, its benefit in sleep quality remains unclear. Nonetheless, PR is beneficial in improving skeletal and respiratory muscle strength, ${ }^{21,23}$ reduced dynamic hyperinflation, ${ }^{24}$ and adaptations in breathing patterns. ${ }^{25}$ Thus, PR may be considered beneficial to the sleep quality of patients with COPD.

Unfortunately, there are very few studies regarding PR and sleep quality in patients with COPD. The present study aims to investigate the benefits of PR in sleep quality in COPD. The primary objective was to determine the effect of PR on subjective sleep quality. The secondary objective was to determine the benefits of PR on HRQOL and exercise capacity.

\section{QUICK LOOK}

\section{Current knowledge}

Pulmonary rehabilitation (PR) is a comprehensive intervention including education, training, and behavior modification aimed at improving quality of life in patients with chronic respiratory disease. The impact of PR on sleep quality is not well described.

\section{What this paper contributes to our knowledge}

In a small group of subjects with COPD, a 12-week session of PR was associated with improvements in sleep quality as measured by a sleep questionnaire. Health-related quality of life and dyspnea were improved after PR.

\section{Methods}

\section{Patient Selection}

From May 2011 to November 2012, 34 subjects were recruited from the out-patient clinic of the Taipei Tzu Chi Hospital. The inclusion criteria were (1) a diagnosis of COPD based on the GOLD guidelines ${ }^{26}$; (2) stability with no exacerbations or worsening of respiratory symptoms, no increased use of rescue medication, and no unscheduled visits for at least 3 months ${ }^{27}$; and (3) ability to mobilize independently. The exclusion criteria were (1) history of other lung diseases such as pneumoconiosis, bronchiectasis, pulmonary tuberculosis, primary pulmonary hypertension, pulmonary embolism, and interstitial lung disease and (2) orthopedic, neurologic, or cardiovascular impairment that might render the subjects incapable of completing the exercise training. The research protocol was approved by the ethics committee of the Taipei Tzu Chi Hospital, and all subjects provided informed consent.

\section{Measurements}

Physiologic parameters were assessed by spirometry, respiratory muscle strength testing (maximum inspiratory pressure $\left[\mathrm{P}_{\mathrm{I}_{\max }}\right]$ and maximum expiratory pressure $\left[\mathrm{P}_{\mathrm{E}_{\max }}\right]$ ), and cardiopulmonary exercise testing. Sleep quality was assessed by the PSQI. The HRQOL and dyspnea symptoms were assessed using the St George Respiratory Questionnaire (SGRQ) and the Borg dyspnea scale. These assessments were performed before and after PR. The physician and technician who performed or interpreted these pre-PR and post-PR intervention measurements were not involved in the trial and were blinded to this study. 


\section{Sleep Quality Assessment}

The validated Chinese version of the PSQI was used to measure sleep quality. ${ }^{28,29}$ The scale is composed of 19 items divided into 7 components: sleep quality, latency, duration, disturbances, habitual sleep efficiency, use of sleep medications, and daytime dysfunction. Each item is rated on a scale of $0-3$, where zero indicates no difficulty, and 3 indicates severe difficulty. A global sleep quality index was calculated with scores ranging from 0 and 21.28,29 Higher PSQI scores represent worse sleep quality, and a PSQI score $>5$ indicates poor sleep quality. ${ }^{28,29}$

\section{Health-Related Quality of Life Assessment}

The HRQOL was assessed using the validated Chinese version of the SGRQ, ${ }^{30}$ which is a questionnaire designed to measure the influence of chest diseases on HRQOL. ${ }^{30}$ Responses to its 50 items were aggregated into an overall score and 3 subscores for symptoms ( 8 items), activity (16 items), and impact (26 items). Responses were weighted, and scores were calculated by dividing the summed weights by the maximum possible weight, with zero as the best possible score and 100 the worst. ${ }^{30}$

\section{Pulmonary Function Test}

Pulmonary function testing for $\mathrm{FEV}_{1}$ and $\mathrm{FVC}$ were performed by spirometry (Medical Graphics, Minneapolis, Minnesota) following the standards of the American Thoracic Society and the European Respiratory Society. ${ }^{31,32}$ The best flow-volume loop was used in the final data analysis.

\section{Respiratory Muscle Strength}

The $\mathrm{P}_{\mathrm{I}_{\max }}$ and $\mathrm{P}_{\mathrm{E}_{\max }}$ were assessed using a standard mouthpiece and direct-dial pressure gauge (respiratory pressure meter, CareFusion, San Diego, California). The $\mathrm{P}_{\mathrm{I}_{\max }}$ was measured at residual volume as the subjects inhaled as hard and as quickly as possible. The $\mathrm{P}_{\mathrm{E}_{\max }}$ was measured at total lung capacity as the subjects exhaled as hard and as quickly as possible. The $\mathrm{P}_{\mathrm{I}_{\max }}$ and $\mathrm{P}_{\mathrm{E}_{\max }}$ were measured several times, and after 4 or 5 attempts, a plateau of values showed relatively little variability $( \pm 10 \%){ }^{33}$

\section{Cardiopulmonary Exercise Test}

The cardiopulmonary exercise test is an incremental symptom-limited exercise test performed on an electronically braked cycle ergometer (Corival, Lode BV, Groningen, The Netherlands). The standard bicycle exercise ramp work load protocol was based on the method of Wasserman et al. ${ }^{34}$ The subjects were strongly encouraged to achieve their point of maximal exercise. Expired air was continuously analyzed using a cardiopulmonary diagnostic system (BreezeSuite 6.1, MGC Diagnostics, St. Paul, Minnesota) to assess physiologic responses to exercise. Oxygen uptake $\left(\dot{\mathrm{V}}_{\mathrm{O}_{2}}\right)$, carbon dioxide output, minute ventilation, breathing frequency, tidal volume, $\mathrm{S}_{\mathrm{pO}_{2}}$, end-tidal $\mathrm{P}_{\mathrm{CO}_{2}}$, electrocardiography, heart rate, and blood pressure were measured continuously during the exercise test. The Borg dyspnea scale was rated at rest and at peak exercise.

\section{Pulmonary Rehabilitation}

All subjects participated in 12-week (2 sessions/week) hospital-based out-patient PR. In each session, formal education, including breathing training, proper use of medications, and self-management skills, was given individually. After education, exercise training with a lower limb cycle ergometer was provided. Each training session was $\sim 40 \mathrm{~min}$ and was closely monitored by a rehabilitation therapist. Work rate, $\mathrm{S}_{\mathrm{pO}_{2}}$, heart rate, blood pressure, Borg dyspnea scale, and leg fatigue during exercise training were monitored.

\section{Statistical Analysis}

Baseline measurements and results after PR were expressed as mean $\pm \mathrm{SD}$. Paired $t$ tests were used to compare measurements before and after PR. Statistical significance was set at $P<.05$. All statistical analyses were performed using SPSS 18.0 (SPSS, Chicago, Illinois). A post hoc power calculation was performed using the $G *$ Power software to strengthen the substantial effect of PR on sleep quality (power $>80 \%$ to reject the null hypothesis with $\alpha=.05)$.

The sample size was estimated based on $\alpha$ level, $\beta$ level, and effect size. ${ }^{35}$ The $\alpha$ level was set as .05 , which indicates the probability of mistakenly rejecting the null hypothesis, and the $\beta$ level was set as .20 , which indicates the probability of mistakenly supporting the null hypothesis. The effect size was set as $0.2-0.5$ using Cohen's d rules of one-sample study design, which indicates a smallto-medium effect of experimental intervention (PR) on the important outcomes (sleep quality). Finally, 34-52 subjects were need to participate in our study. The results indicated that the means \pm SD of the PSQI for pretesting and posttesting were $9.41 \pm 4.33$ and $7.82 \pm 3.90$. The final effect size of PR on sleep quality was 0.41 , which represented a small-to-medium effect size and indicated a substantial effect of PR on sleep quality in patients with COPD. 
Table 1. Baseline Characteristics of Subjects With COPD

\begin{tabular}{|c|c|}
\hline Characteristics & Values \\
\hline Age, mean $\pm \mathrm{SD}, \mathrm{y}$ & $70.2 \pm 9.4$ \\
\hline $\mathrm{BMI}$, mean $\pm \mathrm{SD}, \mathrm{kg} / \mathrm{cm}^{2}$ & $21.9 \pm 3.9$ \\
\hline \multicolumn{2}{|l|}{ Gender, $n(\%)$} \\
\hline Male & $28(82.4)$ \\
\hline Female & $6(17.6)$ \\
\hline \multicolumn{2}{|l|}{ Smoking, $n(\%)$} \\
\hline Current smokers & 7 (20.6) \\
\hline Ex-smokers & $22(64.7)$ \\
\hline Nonsmokers & $5(14.7)$ \\
\hline \multicolumn{2}{|l|}{ Severity of COPD, $n(\%)$} \\
\hline Stage I (mild) & $1(2.9)$ \\
\hline Stage II (moderate) & $16(47.1)$ \\
\hline Stage III (severe) & $13(38.2)$ \\
\hline Stage IV (very severe) & $4(11.8)$ \\
\hline $\mathrm{FEV}_{1} / \mathrm{FVC}$, mean $\pm \mathrm{SD}$ & $0.49 \pm 0.13$ \\
\hline $\mathrm{FEV}_{1}$, mean $\pm \mathrm{SD}, \mathrm{L}$ & $1.06 \pm 0.49$ \\
\hline $\mathrm{FEV}_{1}$, mean $\pm \mathrm{SD}, \%$ of predicted & $49.7 \pm 18.0$ \\
\hline $\mathrm{FVC}$, mean $\pm \mathrm{SD}, \mathrm{L}$ & $2.19 \pm 0.77$ \\
\hline $\mathrm{FVC}, \%$ of predicted & $80.4 \pm 21.8$ \\
\hline $\mathrm{P}_{\mathrm{I}_{\max }}$, mean $\pm \mathrm{SD}, \mathrm{cm} \mathrm{H}_{2} \mathrm{O}$ & $73.4 \pm 27.7$ \\
\hline $\mathrm{P}_{\mathrm{I}_{\max }}^{\max }$, mean $\pm \mathrm{SD}, \%$ & $74.7 \pm 24.4$ \\
\hline $\mathrm{P}_{\mathrm{E}_{\max }}$, mean $\pm \mathrm{SD}, \mathrm{cm} \mathrm{H}_{2} \mathrm{O}$ & $120.3 \pm 38.9$ \\
\hline $\mathrm{P}_{\mathrm{E}_{\max }}^{\max }$, mean $\pm \mathrm{SD}, \%$ & $67.0 \pm 21.7$ \\
\hline \multicolumn{2}{|l|}{$\begin{array}{l}\mathrm{BMI}=\text { body mass index } \\
\mathrm{P}_{\mathrm{I}_{\max }}=\text { maximum inspiratory pressure } \\
\mathrm{P}_{\mathrm{E}_{\max }}=\text { maximum expiratory pressure }\end{array}$} \\
\hline
\end{tabular}

\section{Results}

\section{Anthropometric and Spirometric Data}

The baseline demographic and spirometric data of the subjects with COPD (Table 1) showed that the mean $\mathrm{FEV}_{1} / \mathrm{FVC}$ was $0.49 \pm 0.13$, and the mean $\mathrm{FEV}_{1}$ was $1.06 \pm 0.49 \mathrm{~L} / \mathrm{min}(49.7 \pm 18.0 \%$ of predicted $)$. Most subjects had moderate-to-severe COPD.

\section{Sleep Quality, Disease-Specific HRQOL, and Dyspnea Scores After PR}

PR improved sleep quality (PSQI), HRQOL (SGRQ), and dyspnea scores (Fig. 1). After PR, the mean PSQI decreased from $9.41 \pm 4.33$ to $7.82 \pm 3.90(P<.001)$ (Fig. 1A). In the PSQI, sleep duration, sleep disturbance, and daytime dysfunction showed significant improvement after PR (Fig. 1B). Before PR, a majority of the subjects $(85.3 \%)$ rated sleep as abnormal (PSQI $>5$ ). This decreased to $64.7 \%$ after PR (Fig. 1C). There were also significant improvements in HRQOL and all domains of SGRQ after PR (symptoms, $P=.003$; activity, $P=.03$; impact, $P=.02$; total, $P=.003$ ) (Fig. 2). There was also significant improvement in exertional dyspnea after PR $(P<.001)$ (Fig. 3).

\section{Physiologic Changes in Exercise Capacity, Pulmonary Function, and Respiratory Muscle Strength After PR}

Post-PR changes in exercise capacity, pulmonary function, and respiratory muscle strength were demonstrated. After PR, there were significant improvements in exercise capacity, with increased maximal $\dot{\mathrm{V}}_{\mathrm{O}_{2}}$ (mean increase $72.7 \mathrm{~mL} / \mathrm{min}, P=.004$ ) and work rate (mean increase 9.9 watts, $P<.001$ ) (Fig. 4). There were no significant changes in pulmonary function test results $\left(\mathrm{FEV}_{1}, \mathrm{FVC}\right.$, and $\mathrm{FEV}_{1} / \mathrm{FVC}$ ) after PR (Fig. 5). Respiratory muscle strength significantly improved after $\mathrm{PR}\left(\left[\mathrm{P}_{\mathrm{I}_{\max }}\right], P=.005 ;\left[\mathrm{P}_{\mathrm{E}_{\max }}\right]\right.$, $P=.004)$ (Fig. 6).

\section{Discussion}

This study has 2 major findings. First, the majority of subjects with COPD experienced poor sleep quality. Second, PR improved sleep quality with concurrent improvements of HRQOL, exercise capacity, and respiratory muscle strength. This study also demonstrated that exercise training improved sleep quality, increased sleep duration, and decreased sleep disturbance in subjects with COPD.

Studies on exercise training for improving sleep quality in COPD are limited. Nonetheless, some studies show that exercise training programs improve sleep health in other populations with different diseases and in middle-aged and older adults. ${ }^{36-38}$ Passos et al ${ }^{36}$ suggested that aerobic exercise may reduce presleep anxiety and improve sleep quality in patients with primary insomnia. King et al ${ }^{37}$ evaluated the effect of 4 months of exercise on sedentary individuals with reported sleep complaints and demonstrated that exercise programs improved their self-rated sleep quality. In the present study, an exercise program also had a positive effect on sleep quality in patients with COPD.

Poor sleep quality in patients with COPD is considered a result of multiple contributing factors, including diseasespecific symptoms, concomitant medications, older age, underlying anxiety and depression, and presence of comorbidities. ${ }^{9}$ Respiratory disturbances during sleep have been associated with sleep-related oxygen desaturation, reduction in pulmonary function, and development of hypoventilation. ${ }^{39}$ Hypoventilation during sleep causes significant gas-exchange alteration, resulting in hypercapnia and hypoxia, ${ }^{9}$ which in turn lead to increased sleep arousals and sleep disruption. ${ }^{9}$ Moreover, there is a normal circadian change in airways leading to nocturnal bronchoconstriction with increased airway resistance. ${ }^{9}$ Intercostal muscle activity decreases during sleep, and this is partic- 

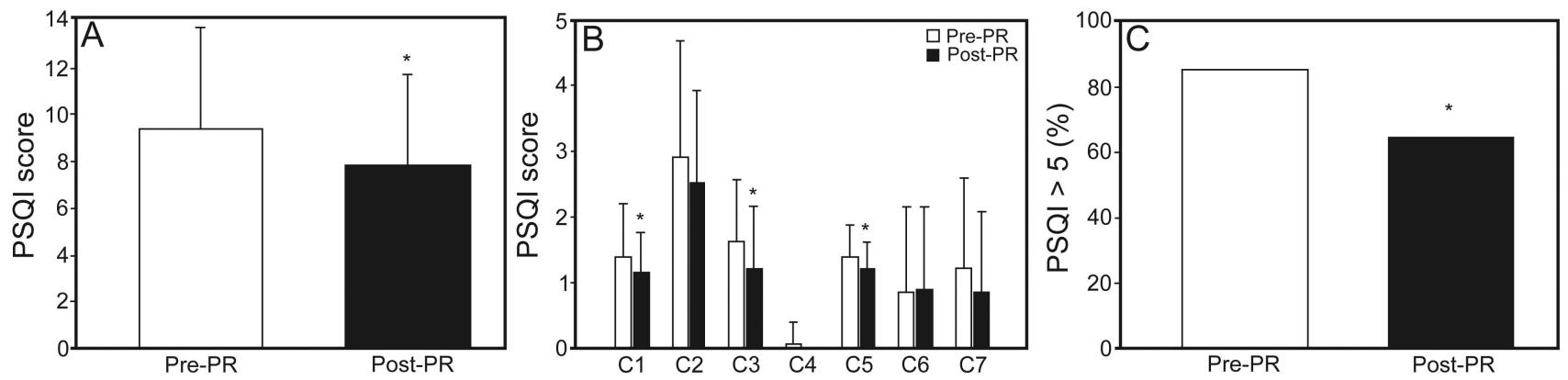

Fig. 1. Scoring and specific items on the Pittsburgh Sleep Quality Index (PSQI). A: After pulmonary rehabilitation (PR), the mean PSQI decreased from $9.41 \pm 4.33$ to $7.82 \pm 3.90(P<.05)$. B: Sleep duration, sleep disturbance, and daytime dysfunction showed significant improvement after PR. C: Before PR, the majority of patients (85.3\%) rated sleep as abnormal (PSQI $>5$ ). The number decreased to $64.7 \%$ after PR. Data are shown as mean \pm SD. * Significant difference from pre-PR $(P<.001)$. C1 = sleep quality; C2 = sleep latency; C3 = sleep duration; C4 = sleep efficiency; C5 = sleep disturbance; C6 = sleep medications; C7 = daytime dysfunction.

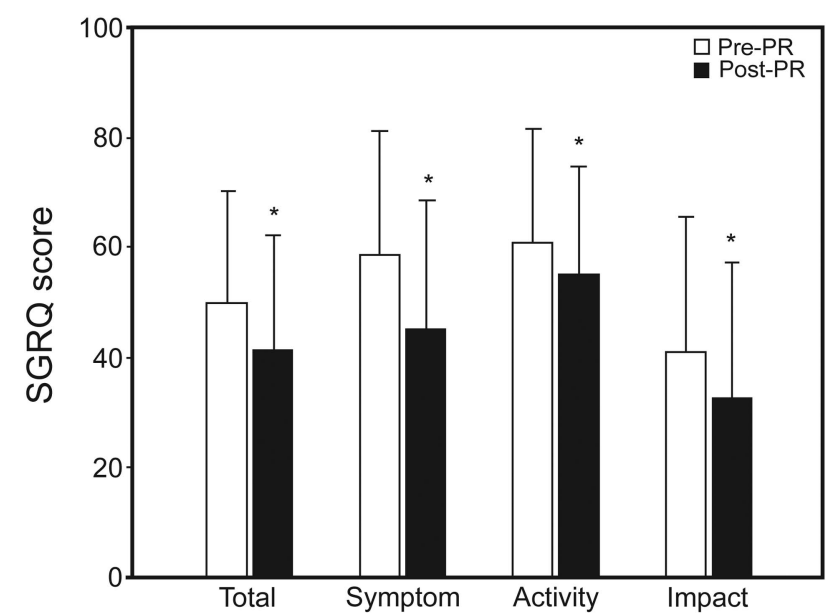

Fig. 2. There were significant improvements in the St George Respiratory Questionnaire (SGRQ): total, symptoms, activity, and impact domains after pulmonary rehabilitation (PR). Data are shown as mean \pm SD. * Significant difference from pre-PR $(P<.001)$.

ularly significant in patients with COPD because they are dependent on accessory muscle activity to maintain ventilation. ${ }^{9}$ The overall physiologic changes in COPD, including nocturnal hypoventilation, air-flow obstruction, hypoxia, hypercapnia, and use of accessory muscles of respiration, lead to poor sleep quality. ${ }^{40}$

Exercise is commonly believed to improve sleep quality. ${ }^{38}$ However, the exact mechanisms of how exercise training improves sleep quality in patients with COPD are unclear. Consideration of the mechanisms underlying the effect of exercise on sleep quality is beyond the scope of this study, but they are believed to consist of a complex set of activities, including physiological and psychological benefits.

Physiologically, PR improves muscle adaptations with increases in aerobic enzymes, further leading to improved respiratory muscle strength. ${ }^{23,41}$ This improvement is important in as much as it is associated with a reduction in

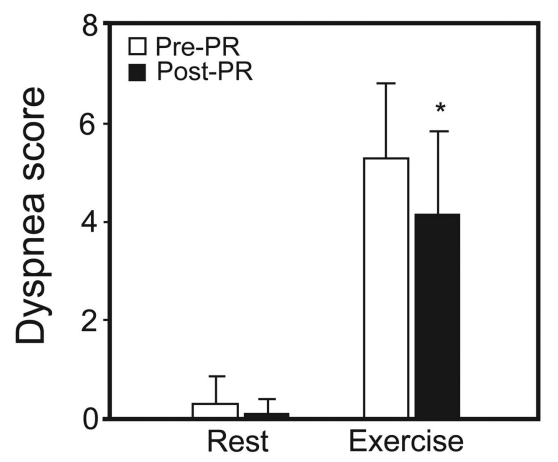

Fig. 3. The Borg dyspnea scale at peak exercise showed significant improvement after pulmonary rehabilitation (PR). Data are shown as mean $\pm S D$. * Significant difference from pre-PR $(P<.001)$.

dynamic hyperinflation ${ }^{23,24}$ and improves ventilation in patients with COPD. In addition, PR also leads to improvement of systemic inflammation. ${ }^{42}$ Pinho et al ${ }^{42}$ suggested that patients with COPD are characterized by increased systemic and pulmonary oxidative stress, and PR was associated with decreased oxidative stress. Exercise training has also been proposed to improve sleep quality by increasing energy consumption, endorphin secretion, and body temperature in a manner that facilitates sleep for body recuperation. ${ }^{38}$ Physical fitness is known to be associated with sleep quality, ${ }^{43}$ and greater improvements in fitness after exercise training are associated with better sleep outcomes. ${ }^{44}$

Psychologically, anxiety and depression can lead to poor sleep quality in patients with COPD. ${ }^{45}$ The prevalence of depression and anxiety is high in COPD. ${ }^{46}$ A meta-analysis of 6 randomized controlled trials concluded that PR is effective for the reduction of anxiety and depression. ${ }^{47} \mathrm{PR}$ leads to better psychological status on the anxiety-depression scale in patients with COPD. ${ }^{20}$ It has been suggested 

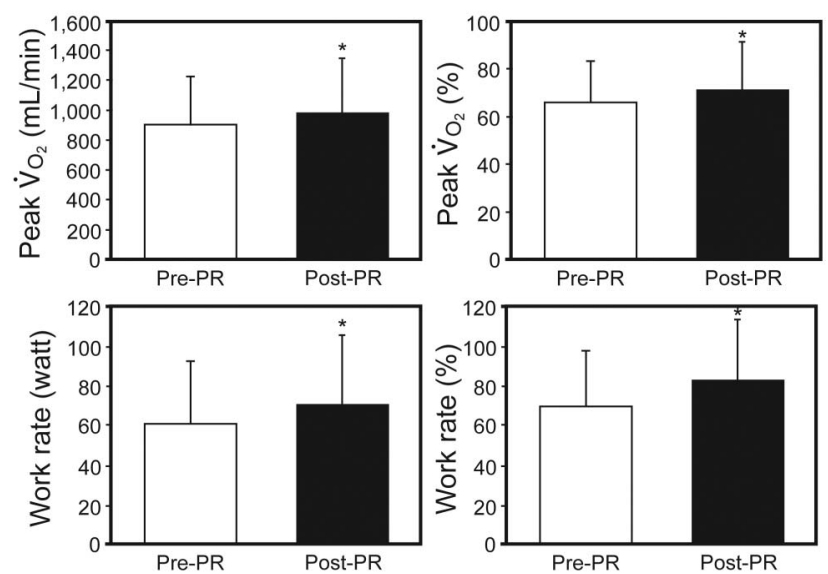

Fig. 4. After pulmonary rehabilitation (PR), there were significant improvements in exercise capacity, with increased maximal oxygen uptake $\left(\dot{\mathrm{V}}_{\mathrm{O}_{2}}\right)$ (mean increase $72.7 \mathrm{~mL} / \mathrm{min}, P=.004 ; 5.2 \%$, $P=.005$ ) and work rate (mean increase 9.9 watts, $P<.001$; $12.7 \%, P<.001)$. Data are shown as mean \pm SD. ${ }^{*}$ Significant difference from pre-PR $(P<.001)$.

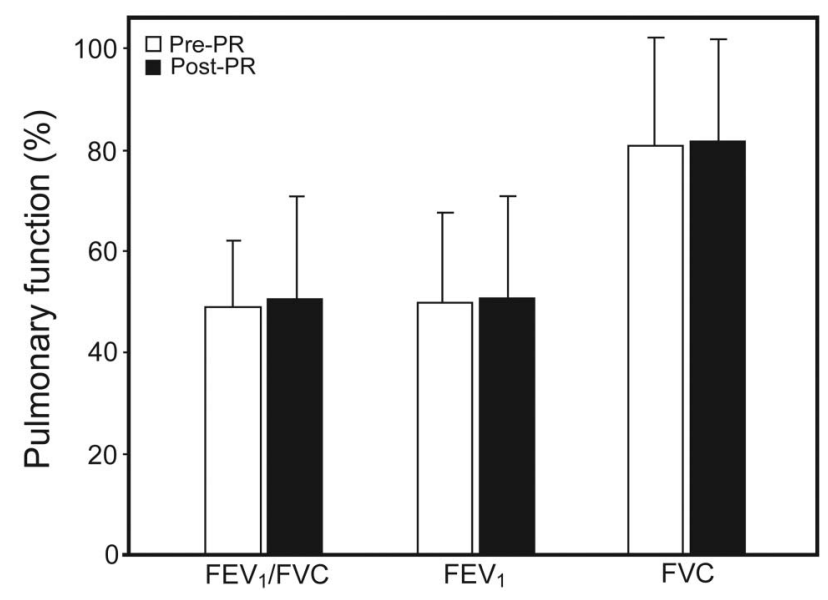

Fig. 5. After pulmonary rehabilitation (PR), there were no significant changes in pulmonary function test results. Data are shown as mean \pm SD. ${ }^{*}$ Significant difference from pre-PR $(P<.001)$.

that exercise training promotes sleep via its anxiolytic or antidepressant effects. ${ }^{48}$

There are multiple improvements resulting from PR in patients with COPD, including physiological and psychological benefits. Although the exact mechanisms of improvement of sleep quality after PR are not fully understood, exercise training could be an effective nonpharmacologic treatment for patients with COPD according to this study.

The present study has some limitations. First, the overlap of obstructive sleep apnea syndrome in patients with COPD may exacerbate poor sleep quality. ${ }^{49}$ In previous studies, the prevalence of overlap syndrome in COPD was $8.6-11 \% .^{50,51}$ Polysomnography was not performed in the

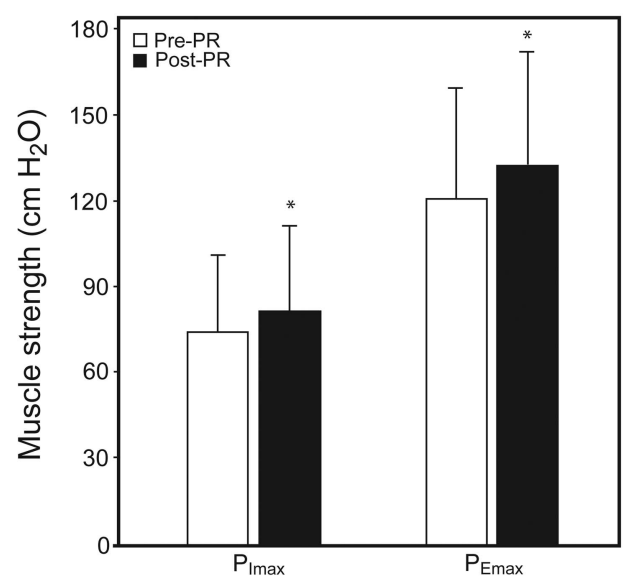

Fig. 6. After pulmonary rehabilitation (PR), there were significant improvements in respiratory muscle strength. Data are shown as mean $\pm \mathrm{SD}$. ${ }^{*}$ Significant difference from pre-PR $(P<.001)$. $P_{I_{\max }}=$ maximum inspiratory pressure. $P_{E_{\max }}=$ maximum expiratory pressure.

current study, and the prevalence of organic sleep disorders in this study population could not be objectively determined. Therefore, it is hard to determine the influence of obstructive sleep apnea syndrome or other organic sleep disorders in this study. Second, nighttime oxygenation saturations were not measured. Nocturnal oxygen desaturation is an important factor contributing to sleep disturbances, but there were no data on nocturnal oxygenation in the study. Future studies on PR and sleep quality should incorporate this important measure. Third, only the shortterm effect of PR on sleep quality was evaluated. It is necessary to perform long-term studies to evaluate the duration of improvement in sleep quality after 3 months of PR.

\section{Conclusions}

Patients with COPD often have poor sleep quality. PR may provide significant improvement in sleep quality, with concurrent improvement in HRQOL and exercise capacity. As such, PR can be recommended as an effective nonpharmacologic treatment to improve sleep quality in patients with COPD, and it should be part of their clinical management.

\section{REFERENCES}

1. Klink M, Quan SF. Prevalence of reported sleep disturbances in a general adult population and their relationship to obstructive airways diseases. Chest 1987;91(4):540-546.

2. Nunes DM, Mota RM, de Pontes Neto OL, Pereira ED, de Bruin VM, de Bruin PF. Impaired sleep reduces quality of life in chronic obstructive pulmonary disease. Lung 2009;187(3):159-163.

3. Lewis CA, Fergusson W, Eaton T, Zeng I, Kolbe J. Isolated nocturnal desaturation in COPD: prevalence and impact on quality of life and sleep. Thorax 2009;64(2):133-138. 


\section{PR ImPRoves SLEEP QUALITY IN COPD}

4. Scharf SM, Maimon N, Simon-Tuval T, Bernhard-Scharf BJ, Reuveni $\mathrm{H}$, Tarasiuk A. Sleep quality predicts quality of life in chronic obstructive pulmonary disease. Int J Chron Obstruct Pulmon Dis 2011;6:1-12.

5. Valipour A, Lavie P, Lothaller H, Mikulic I, Burghuber OC. Sleep profile and symptoms of sleep disorders in patients with stable mild to moderate chronic obstructive pulmonary disease. Sleep Med 2011; 12(4):367-372.

6. Narang I, Manlhiot C, Davies-Shaw J, Gibson D, Chahal N, Stearne $\mathrm{K}$, et al. Sleep disturbance and cardiovascular risk in adolescents. CMAJ 2012;184(17):E913-E920.

7. Wang CY, Chau LY. Heme oxygenase-1 in cardiovascular diseases: molecular mechanisms and clinical perspectives. Chang Gung Med J 2010;33(1):13-24.

8. Cappuccio FP, D'Elia L, Strazzullo P, Miller MA. Quantity and quality of sleep and incidence of type 2 diabetes: a systematic review and meta-analysis. Diabetes Care 2010;33(2):414-420.

9. Gay PC. Chronic obstructive pulmonary disease and sleep. Respir Care 2004;49(1):39-51; discussion 51-52.

10. Ryan S, Doherty LS, Rock C, Nolan GM, McNicholas WT. Effects of salmeterol on sleeping oxygen saturation in chronic obstructive pulmonary disease. Respiration 2010;79(6):475-481.

11. Martin RJ, Bartelson BL, Smith P, Hudgel DW, Lewis D, Pohl G, et al. Effect of ipratropium bromide treatment on oxygen saturation and sleep quality in COPD. Chest 1999;115(5):1338-1345.

12. Mulloy E, McNicholas WT. Theophylline improves gas exchange during rest, exercise, and sleep in severe chronic obstructive pulmonary disease. Am Rev Respir Dis 1993;148(4 Pt 1):1030-1036.

13. McNicholas WT, Calverley PM, Lee A, Edwards JC. Long-acting inhaled anticholinergic therapy improves sleeping oxygen saturation in COPD. Eur Respir J 2004;23(6):825-831.

14. McKeon JL, Murree-Allen K, Saunders NA. Supplemental oxygen and quality of sleep in patients with chronic obstructive lung disease. Thorax 1989;44(3):184-188

15. Orth M, Walther JW, Yalzin S, Bauer TT, de Zeeuw J, Kotterba S, et al. Influence of nocturnal oxygen therapy on quality of life in patients with COPD and isolated sleep-related hypoxemia: a prospective, placebo-controlled cross-over trial. Pneumologie 2008; 62(1): $11-16$

16. Agusti A, Hedner J, Marin JM, Barbé F, Cazzola M, Rennard S. Night-time symptoms: a forgotten dimension of COPD. Eur Respir Rev 2011;20(121):183-194.

17. Paudel ML, Taylor BC, Diem SJ, Stone KL, Ancoli-Israel S, Redline $\mathrm{S}$, et al. Association between depressive symptoms and sleep disturbances in community-dwelling older men. J Am Geriatr Soc 2008; 56(7):1228-1235.

18. Ries AL, Bauldoff GS, Carlin BW, Casaburi R, Emery CF, Mahler DA, et al. Pulmonary rehabilitation: joint ACCP/AACVPR evidencebased clinical practice guidelines. Chest 2007;131(5 Suppl):4S-42S

19. Nici L, Donner C, Wouters E, Zuwallack R, Ambrosino N, Bourbeau J, et al. American Thoracic Society/European Respiratory Society statement on pulmonary rehabilitation. Am J Respir Crit Care Med 2006;173(12):1390-1413

20. Ergün P, Kaymaz D, Günay E, Erdoğan Y, Turay UY, Demir N, et al. Comprehensive out-patient pulmonary rehabilitation: treatment outcomes in early and late stages of chronic obstructive pulmonary disease. Ann Thorac Med 2011;6(2):70-76.

21. Lan CC, Chu WH, Yang MC, Lee CH, Wu YK, Wu CP. Benefits of pulmonary rehabilitation in patients with COPD with normal exercise capacity. Respir Care 2013;58(9):1482-1488.

22. Lan CC, Yang MC, Lee CH, Huang YC, Huang CY, Huang KL, Wu YK. Pulmonary rehabilitation improves exercise capacity and quality of life in underweight patients with chronic obstructive pulmonary disease. Respirology 2011;16(2):276-283.
23. Vogiatzis I, Stratakos G, Simoes DC, Terzis G, Georgiadou O, Roussos C, Zakynthinos S. Effects of rehabilitative exercise on peripheral muscle TNF-alpha, IL-6, IGF-I and MyoD expression in patients with COPD. Thorax 2007;62(11):950-956.

24. Casaburi R, Porszasz J, Burns MR, Carithers ER, Chang RS, Cooper CB. Physiologic benefits of exercise training in rehabilitation of patients with severe chronic obstructive pulmonary disease. Am J Respir Crit Care Med 1997;155(5):1541-1551.

25. Gigliotti F, Coli C, Bianchi R, Romagnoli I, Lanini B, Binazzi B, Scano G. Exercise training improves exertional dyspnea in patients with COPD: evidence of the role of mechanical factors. Chest 2003; 123(6):1794-1802.

26. Global Initiative for Chronic Obstructive Lung Disease. Global strategy for the diagnosis, management, and prevention of chronic pulmonary disease. Updated 2011. http://www.goldcopd.com/down load.asp?intId=504. Accessed November 20, 2013.

27. Burge S, Wedzicha JA. COPD exacerbations: definitions and classifications. Eur Respir J Suppl 2003;41:46s-53s.

28. Chan SF, Chen TH, Liao YM, Chou KR, Tsai PS. Development and preliminary validation of the Chinese version of the Sleep-Associated Monitoring Index. Int J Nurs Stud 2012;49(1):54-64.

29. Buysse DJ, Reynolds CF 3rd, Monk TH, Berman SR, Kupfer DJ. The Pittsburgh Sleep Quality Index: a new instrument for psychiatric practice and research. Psychiatry Res 1989;28(2):193-213.

30. Wang KY, Chiang CH, Maa SH, Shau WY, Tarn YH. Psychometric assessment of the Chinese language version of the St. George's Respiratory Questionnaire in Taiwanese patients with bronchial asthma. J Formos Med Assoc 2001;100(7):455-460.

31. American Thoracic Society. Standardization of spirometry. Am J Respir Crit Care Med 1995;152(3):1107-1136.

32. Miller MR, Crapo R, Hankinson J, Brusasco V, Burgos F, Casaburi $\mathrm{R}$, et al. General considerations for lung function testing. Eur Respir J 2005;26(1):153-161.

33. McConnell AK, Copestake AJ. Maximum static respiratory pressures in healthy elderly men and women: issues of reproducibility and interpretation. Respiration 1999;66(3):251-258.

34. Wasserman K, Hansen JE, Sue DY, Stringer WW, Whipp BJ. Principles of exercise testing and interpretation: including pathophysiology and clinical applications. Philadelphia: Lippincott Williams \& Wilkins; 2005:10-65.

35. Cohen J. Statistical power analysis for the behavioral sciences, 2nd edition. Hillsdale, NJ: Lawrence Erlbaum Associates; 1988:273-406.

36. Passos GS, Poyares D, Santana MG, Garbuio SA, Tufik S, Mello MT. Effect of acute physical exercise on patients with chronic primary insomnia. J Clin Sleep Med 2010;6(3):270-275.

37. King AC, Oman RF, Brassington GS, Bliwise DL, Haskell WL. Moderate-intensity exercise and self-rated quality of sleep in older adults. A randomized controlled trial. JAMA 1997;277(1):32-37.

38. Yang PY, Ho KH, Chen HC, Chien MY. Exercise training improves sleep quality in middle-aged and older adults with sleep problems: a systematic review. J Physiother 2012;58(3):157-163.

39. Collop N. Sleep and sleep disorders in chronic obstructive pulmonary disease. Respiration 2010;80(1):78-86.

40. Krachman S, Minai OA, Scharf SM. Sleep abnormalities and treatment in emphysema. Proc Am Thorac Soc 2008;5(4):536-542.

41. Muñoz Marín D, Olcina G, Timón R, Robles MC, Caballero MJ, Maynar M. Effect of different exercise intensities on oxidative stress markers and antioxidant response in trained cyclists. J Sports Med Phys Fitness 2010;50(1):93-98.

42. Pinho RA, Chiesa D, Mezzomo KM, Andrades ME, Bonatto F, Gelain D, et al. Oxidative stress in chronic obstructive pulmonary disease patients submitted to a rehabilitation program. Respir Med 2007;101(8):1830-1835. 


\section{PR ImPRoves SLEEP QuAlity IN COPD}

43. Costigan SA, Barnett L, Plotnikoff RC, Lubans DR. The health indicators associated with screen-based sedentary behavior among adolescent girls: a systematic review. J Adolesc Health 2013;52(4): 382-392.

44. Tworoger SS, Yasui Y, Vitiello MV, Schwartz RS, Ulrich CM, Aiello EJ, et al. Effects of a yearlong moderate-intensity exercise and a stretching intervention on sleep quality in postmenopausal women. Sleep 2003;26(7):830-836.

45. Suh S, Ellis RJ, Sollers JJ 3rd, Thayer JF, Yang HC, Emery CF. The effect of anxiety on heart rate variability, depression, and sleep in chronic obstructive pulmonary disease. J Psychosom Res 2013;74(5):407-413.

46. Yohannes AM, Willgoss TG, Baldwin RC, Connolly MJ. Depression and anxiety in chronic heart failure and chronic obstructive pulmonary disease: prevalence, relevance, clinical implications and management principles. Int J Geriatr Psychiatry 2010;25(12):1209-1221.

47. Coventry PA, Hind D. Comprehensive pulmonary rehabilitation for anxiety and depression in adults with chronic obstructive pulmonary disease: systematic review and meta-analysis. J Psychosom Res 2007; 63(5):551-565

48. Passos GS, Poyares D, Santana MG, D'Aurea CV, Youngstedt SD, Tufik S, de Mello MT. Effects of moderate aerobic exercise training on chronic primary insomnia. Sleep Med 2011;12(10):10181027.

49. Shiina K, Tomiyama H, Takata Y, Yoshida M, Kato K, Nishihata Y, et al. Overlap syndrome: additive effects of COPD on the cardiovascular damages in patients with OSA. Respir Med 2012;106(9): 1335-1341.

50. Zanchet RC, Viegas CAA, Lima TSM. [Influence of pulmonary rehabilitation on the sleep patterns of patients with chronic obstructive pulmonary disease]. J Bras Pneumol 2004;30(5):439-444. Article in Portuguese.

51. Chaouat A, Weitzenblum E, Krieger J, Ifoundza T, Oswald M, Kessler R. Association of chronic obstructive pulmonary disease and sleep apnea syndrome. Am J Respir Crit Care Med 1995;151(1):82-86. 